\title{
Effect of Musical Hearing On Anxiety, Pain, and Cardiorespiratory Parameters in Cardiac Surgery: Study Protocol of a Randomized Clinical Trial
}

Érica Vieira de Andrade ( $\square$ erica.andrade860@gmail.com )

Universidade Federal do Triangulo Mineiro https://orcid.org/0000-0002-1813-0925

Vanderlei José Haas

Universidade Federal do Triangulo Mineiro

Maíla Fidalgo de Faria

Universidade Federal do Triangulo Mineiro

Márcia Marques dos Santos Felix

Universidade Federal do Triangulo Mineiro

Maria Beatriz Guimarães Ferreira

Universidade Federal do Triangulo Mineiro

Elizabeth Barichello

Universidade Federal do Triangulo Mineiro

Patricia da Silva Pires

Universidade Federal da Bahia

Maria Helena Barbosa

Universidade Federal do Triangulo Mineiro

\section{Research Article}

Keywords: Anxiety, Pain Postoperative, Cardiac Surgery, Music, Complementary Therapies

Posted Date: August 19th, 2021

DOI: https://doi.org/10.21203/rs.3.rs-396025/v1

License: (c) (i) This work is licensed under a Creative Commons Attribution 4.0 International License.

Read Full License 


\section{Abstract \\ Background}

Preoperative anxiety and postoperative pain are frequent in cardiac surgeries and constitute important stressors for the patient, which can cause several complications. Among the various strategies that aim to alleviate these phenomena is musical hearing as a non-pharmacological intervention. The aim of this study is to evaluate the effect of musical hearing on preoperative state-anxiety, postoperative pain, at rest and cough stimulus, and cardiorespiratory parameters in patients undergoing cardiac surgery.

\section{Methods}

A randomized, parallel, simple masking clinical trial will be conducted with patients 18 years of age or older who have undergone elective cardiac surgery by sternotomy, who accept to participate in the research and sign the consent. Study participants will be randomly divided, in a 1:1 ratio, to one of the two groups, experimental (subjected to music listening for 20 minutes in the pre and postoperative period) or control (standard care in the pre and post -operative), using a randomization scheme generated by the website. The sample size calculation was obtained after conducting a pilot study.

\section{Discussion}

The results of the study may contribute to the implementation of non-pharmacological interventions in health services, highlighting the protocols of musical hearing, to minimize anxiety and pain in cardiac surgery.

\section{Trial registration:}

ReBEC RBR-8mdyhd. Posted on December 10, 2019, https://ensaiosclinicos.gov.br/rg/RBR-8mdyhd

\section{Background}

Cardiac surgery is the treatment of choice for many patients with cardiovascular diseases, which are among the main causes of morbidity and mortality in developed and developing countries. For patients who undergo this surgery, in addition to the pathophysiological aspects, there is also an emotional impact, since the complexity of the procedure and its critical condition can cause anxiety, stress and depression [1].

Therefore, the psychological component must be inserted in an effective preoperative preparation, highlighting the performance and the most active intervention of the nursing team. The preoperative evaluation is essential, starting with the contact between nurse/patient and continuing throughout the 
surgical process. It must be a complete, holistic assessment, which will result in the formulation of nursing diagnoses and intervention decisions [2].

Anxiety is the most common emotion before cardiac surgeries, making up one of the nursing diagnoses and the nurse's preoperative visit is an indispensable procedure, which helps in the psychological preparation of the patient and outlines strategies for the success of the procedure and less complications in the intra and postoperative [3].

Of all types of surgeries, the cardiac is the least psychologically accepted by patients, generating high levels of anxiety in the preoperative period, possibly due to the cultural association of the heart as an organ related to life, death and the generation of feelings. The symbolic consideration of the heart and the fear associated with death lead to emotional distress in patients, which can cause complications in the postoperative period [4].

Studies have evaluated the correlation between preoperative anxiety and postoperative pain in several surgical procedures [5-7]. In the postoperative period of cardiac surgeries, which are complex and large procedures, pain has still been shown to be very frequent, as indicated by several studies [8-13]. It is evident that uncontrolled postoperative pain prevents functional recovery, decreases the patient's quality of life, becomes a risk factor for persistent postoperative pain, chronic pain and disability, in addition to evidencing a low quality of care [14].

Currently, the use of multimodal analgesia is recommended to ensure greater efficacy in relieving postoperative pain when compared to single-mode interventions [15]. Among non-pharmacological therapies, which are also covered in multimodal analgesia, music that has been used in multiple contexts and scenarios of health care stands out.

Several studies conducted with different objectives, evaluated the effects of musical intervention on pain and anxiety in surgery of patients with breast cancer [16], on stress and anxiety in varicose vein surgery [17], on anxiety and hemodynamic parameters during coronary angioplasty [18], in the anxiety of children undergoing elective surgery [19], in the perception of relaxation, anxiety and pain in patients during shockwave lithotripsy [20], in the anxiety and pain during the dressing changes of burned patients [21] among others.

In the context of cardiac surgeries, research that analysed the effects of musical intervention showed conflicting results. Research in Turkey has shown that pain after open heart surgery has decreased in patients who have heard music compared to those who have not. Oxygen saturation also increased significantly within the musical group, but there was no difference in the other physiological parameters [22]. In a survey conducted in Iran, there was also a significant reduction in the intensity of postoperative pain in cardiac surgery among patients who heard music [23].

However, for other authors [24], music can reduce pain and anxiety in patients with heart disease, but in general, the results on the effects of music are still inconsistent. Other researchers also concluded, in their 
review, that after cardiac surgery, currently, there is still insufficient evidence to recommend music as an effective non-pharmacological option to reduce pain and anxiety, requiring further studies and standardized intervention protocols [25].

In light of the above, the relevance of this research is observed in terms of minimizing gaps and adding scientific knowledge to the specific area. It is also worth highlighting the importance of evaluating the repercussion of musical intervention in postoperative pain, not only at rest, but also when coughing, as this is an important activity in the prevention of complications after cardiac surgery and which should be encouraged in postoperative.

The hypothesis to be tested in this clinical trial is: Musical hearing in patients undergoing cardiac surgery will promote: a reduction in the average of anxiety-state scores in the immediate preoperative period; reduction of the average of the intensity scores of the pain, at rest and to the cough stimulus, on the 1st postoperative day; reduction in mean systolic and diastolic blood pressure, heart rate, respiratory rate and increase in mean oxygen saturation in the immediate preoperative period and on the first postoperative day.

Therefore, this study has the general objective of evaluating the effect of musical hearing on preoperative anxiety-state, postoperative pain, at rest and cough stimulus, and cardiorespiratory parameters in patients undergoing cardiac surgery.

\section{Methods}

\section{Objectives}

The specific objectives of the clinical trial are:

1. Compare anxiety-state scores between the experimental and control groups in the immediate preoperative period of patients undergoing cardiac surgery;

2. Measure the intensity of acute pain, at rest and cough stimulus, on the 1st postoperative day and identify its location in patients undergoing cardiac surgery;

3. Compare pain intensity scores, at rest and cough stimulus, between the experimental and control groups, on the 1st postoperative day of patients undergoing cardiac surgery;

4. Compare cardiorespiratory parameters (blood pressure, heart rate, respiratory rate and oxygen saturation), between the experimental and control groups, in the immediate preoperative period and on the 1st postoperative day of patients undergoing cardiac surgery;

5. Compare intra-group, preoperative anxiety-state scores, postoperative pain scores, at rest and cough stimulus, and cardiorespiratory parameters (blood pressure, heart rate, respiratory rate and oxygen saturation) in patients undergoing cardiac surgery;

6. To verify the correlation between preoperative anxiety-state and intensity of postoperative pain in patients undergoing cardiac surgery. 


\section{Study design}

The study will be a single-centred, randomized, parallel clinical trial with simple masking and will follow the Consolidated Standards of Reporting Trials (CONSORT) Declaration - extension for randomized clinical trials of non-pharmacological treatments [26].

This clinical trial protocol has been designed in accordance with the Standard Protocol Items: Recommendations for Interventional Trials (SPIRIT) guideline [27], as evidenced in the checklist (see Additional File 1).

\section{Study location}

The study will be developed at the Surgical Clinic Unit and Coronary Intensive Care Unit (ICU) of the Hospital de Clínicas of the Federal University of Triângulo Mineiro (HC-UFTM), located in Uberaba, Minas Gerais, Brazil.

\section{Inclusion and exclusion criteria for participants}

The study will include patients who will undergo elective cardiac surgery, aged 18 or over, of both sexes.

Patients with hearing and/or cognitive impairment or impairment (recorded in medical records) will be excluded; patients who are using sedative and/or anxiolytic drugs at the time of data collection, in the immediate preoperative period, and those who are unable to maintain a dialogue with the researcher to answer the items of the data collection instrument.

After meeting the established criteria, patients who express agreement to participate in the research, after all clarifications, will sign the Free and Informed Consent Form.

\section{Randomization, concealment of allocation and masking}

Study participants will be randomly allocated, in a 1: 1 ratio, to one of the groups, experimental (with musical listening) or control (without musical listening), using a randomization scheme generated by a specific website for this purpose.

After randomization, a list will be generated, numbered sequentially, for the allocation of participants to the research groups. To ensure concealment of the allocation, the group of each participant, according to the generated list, will be inserted in opaque envelopes, numbered sequentially, which will be sealed, stored in a safe place, and will only be opened by the researcher responsible for the intervention, at the moment the application of musical listening or standard care for the corresponding participant. The entire process of randomization and concealment of the allocation will be carried out by the statistician without clinical involvement in the study.

The clinical trial will be conducted with simple masking, since the researchers responsible for applying the data collection instrument and measuring the variables studied, as well as the team of prescribers of 
the service will be masked as to the allocation group of each patient. However, due to the nature of the intervention, it will not be possible to mask the research participant, considering that he/she will be aware of being part of the experimental group, as he/she is submitted to musical listening.

\section{Intervention}

The non-pharmacological intervention to be evaluated will be music listening for 20 minutes.

The international research and development organization Joanna Briggs Institute synthesized the best available evidence on the use of music as a therapeutic intervention for the management of anxiety or pain related to surgical interventions and recommends the use of light and non-lyrical music, with 60 to 80 beats per minute, in low tones, preferably string, with minimal percussion and a volume of $60 \mathrm{~dB}$ [28].

The songs selected for this research, as well as their order, comprised part of the collection used in a clinical trial on anxiety in blood donors [29], which had technical advice from two music teachers.

The repertoire, which totals 20 minutes and 28 seconds, consists of five classical and instrumental music from the Baroque, classical and romantic periods, composed between the 18th and 19th centuries. The songs are:

Piece of music 1: Cello Suite nº 1, Prelude / Composer: Johann Sebastian Bach (1685-1750) / Instrument: Cello

Piece of music 2: Nocturne Op. 9 n 2 / Composer: Frédéric Chopin (1810-1849) / Instrument: piano

Piece of music 3: Clarinet Concerto In A Major K 622 Adagio / Composer: Wolfgang Amadeus Mozart (1756-1791)

Piece of music 4: Gymnopédie nº 1 / Composer: Erik Satie (1866-1925) / Instrument: piano

Piece of music 5: The Carnival of the Animals - Le Cygne / Composer: Charles-Camille Saint-Saëns (18351921) / Instruments: piano and cello

The sequence of the songs considered the proposal of initially having selections with more active and faster elements, combining with the level of activity and stress of the listener, and gradually moving on to slower, less stimulating and more relaxing songs [30].

Classical songs have common elements of relaxing and sedative music, so they are often used in recording for relaxation [30].

\section{Experimental group}

The patients in the experimental group will undergo musical hearing in the immediate preoperative period (up to 24 hours before surgery) and on the first postoperative day (24 to 48 hours after surgery), totalling two musical auditions for each patient. 
Each musical audition will have a duration of 20 minutes and will be performed through headphones with a memory card containing the musical recording. The intervention will be conducted by the researcher, who adjusts the volume of the headset, instructs the patient to stay in a comfortable position on the bed, with his eyes closed, and then start listening to classical music. The time of the musical audition will be timed by the researcher.

\section{Control group}

Control group patients will receive standard care in the immediate preoperative period and on the 1st postoperative day. Standard care will consist of bed rest/armchair with routine care, for 20 minutes, timed by the researcher.

\section{Criterion for discontinuing the intervention}

The intervention of a given participant will only be discontinued upon request, withdrawing consent to participate in the research.

\section{Concurrent care}

The study will not interfere with the pharmacological strategy and care of patients, who will receive medications and procedures according to the medical prescription protocol of the study field institution. Information related to painkillers administered to participants will be inserted in the data collection instrument.

\section{Data collection and management}

Data collection will be performed on the patient's bed, once a day, in the immediate preoperative period (up to 24 hours before surgery) and on the first postoperative day (24 to 48 hours after surgery). The data related to the anaesthetic-surgical procedure will be collected from the patient's medical record.

No data will be collected in the immediate postoperative period (first 24 hours after surgery), since in this period, patients may still be under residual anaesthetic effect, making data collection unfeasible.

\section{Data collection instrument}

The data collection instrument, developed specifically for this study, was submitted to content validation by five judges with a doctorate, relevant experience in the field of nursing, teaching and evidence-based research.

The instrument contains four parts:

Part I - identification, sociodemographic and clinical data: initials of the patient's name, medical record number, date of birth, age, sex, ethnicity, origin, education, presented comorbidities, previous cardiac 
surgery and classification of physical status according to the American Society of Anaesthesiologists [31].

Part II - refers to the evaluation in the immediate preoperative period: preoperative time and the following data will be collected before and after the musical intervention or standard care: anxiety-state score according to the State-Trait Anxiety Inventory (STAI) - STAI State, heart rate, systolic and diastolic blood pressure, respiratory rate, and oxygen saturation.

Part III - data regarding the anaesthetic-surgical procedure, to be obtained from the patient's record: surgery performed, anaesthetic block, duration of general anaesthesia, use of cardiopulmonary bypass, duration of cardiopulmonary bypass, duration of surgery, duration of the anaesthetic-surgical procedure, complications in the operating room and placement of a catheter for postoperative analgesia.

Part IV - refers to the evaluation on the 1st postoperative day. Information on postoperative time, extubation time, presence of chest drains and prescribed analgesic regimen will be obtained. After applying the Ramsay sedation scale, if the score is $\leq 3$ points, the following data will be collected before and after the musical intervention or standard care:

- Evaluation of postoperative pain, with the patient at rest and after coughing stimulus: presence of pain, location of pain, pain intensity according to the Numerical Scale and classification of pain intensity;

- Values of heart rate, systolic and diastolic blood pressure, respiratory rate and oxygen saturation.

To assess state-anxiety, the STAI will be used, prepared by Spielberger, Gorsuch \& Lushene [32], translated and validated for Brazilian Portuguese [33]. It consists of two distinct scales that measure two different concepts of anxiety: state of anxiety and trait of anxiety. The STAI anxiety trait scale contains 20 statements that require individuals to describe how they generally feel. The anxiety state scale (STAI State) also consists of 20 statements, but individuals indicate how they feel at a given moment [34].

For each statement, whose weight varies from one to four points, the individual must indicate how he feels, indicating one of the four available alternatives, totalling scores ranging from 20 to 80 points, in each scale [34].

The present research will evaluate only the state anxiety since the objective is to evaluate the level of anxiety in the moment before the surgery.

When applying the scale, it should be made clear to the patient that the questions do not have right or wrong answers and that the answers must correspond to one of the options given [35]. In the case of STAI State, the answer options are: absolutely no $=1$; a little $=2$; quite $=3$; very much $=4$.

To quantify the total score, the sum must be performed, assigning the score for the answer given to each question, however, for positive questions, the score must be inverted, that is, if the patient answers 1 , a value is assigned 4; if you answer 2, a value of 3 is assigned; if you answer 3, you assign a value of 2 and 
if you answer 4, you assign a value of 1 . On the STAI Status scale, the positive questions that should be scored in reverse are: $1,2,5,8,10,11,15,16,19,20$ [35].

It was evident that the scores on the state-anxiety scale increase in response to various types of tension and decrease as a result of relaxation training [34].

As for cardiorespiratory parameters, a portable pulse oximeter will be used to measure heart rate and oxygen saturation; to check the respiratory rate, breathing movements will be observed and counted for one minute. And the measurement of systolic and diastolic blood pressure will be performed by the indirect method of measurement with auscultatory technique, using a stethoscope and calibrated adult aneroid sphygmomanometer. The blood pressure measurement technique will follow the recommendations of the Brazilian Guidelines on Hypertension [36].

To quantify the intensity of postoperative pain, the Numerical Scale of 11 points will be used, graded from 0 to 10, where 0 means no pain and 10 means the worst pain ever felt. This is one of the validated scales for assessing pain intensity [15]. The Numeric Scale is a one-dimensional instrument, which quantifies the severity or intensity of pain, often used because it is quick and easy to apply [37].

Pain intensity will then be classified as: no pain ( 0 ), mild pain (1 to 4), moderate pain (5 to 7 ) and severe pain (8 to 10) [38].

\section{Recruitment and Data Collection Procedures}

To carry out the data collection, a team formed by three nurse researchers linked to the Study and Research Group on Evidence-Based Practice and Patient Safety at UFTM will be constituted.

One of the researchers will recruit the participants, make the allocation, and conduct the musical intervention or time the standard care time. The other researchers will be responsible for collecting the information in the medical records and for applying the data collection instrument to the patients, before and after the intervention or standard care, in the pre and postoperative period.

Before the start of data collection, the team will carry out training on the whole process and the instruments to be used, to guarantee the standardization and the correct execution of the procedures.

For recruitment, initially the researcher will obtain, each week, from the Cardiovascular Unit of HC-UFTM, the identification of the patients who will be submitted to the cardiac surgical procedure, the date of hospitalization and the surgery.

To encourage the recruitment of participants in order to obtain the target sample size, the research will have the support of the institution field of study, as well as the motivational aspect of potential participants, who will be informed about the relevance of the research and the need of their involvement so that scientific advances happen. 
In the immediate preoperative period, in the hospital ward, patients who meet the inclusion criteria will be informed about the research and those who agree to participate, after all clarifications, will sign the Free and Informed Consent Term in two copies, one for the researcher and another for the patient.

Afterwards, the identification, sociodemographic and clinical data of the patients will be collected, followed by the assessment of the state-anxiety level, applying the State STAI and the measurement of the cardiorespiratory parameters (heart rate, systolic and diastolic blood pressure, respiratory rate and oxygen saturation).

Then the intervention will be applied (music listening for 20 minutes) for patients in the experimental group or standard care (routine care) for patients in the control group.

After the intervention or standard care, the instruments for assessing the state-anxiety level and cardiorespiratory parameters will be applied again.

On the 1 st postoperative day, in the Coronary ICU, data related to the anaesthetic-surgical procedure and information on postoperative time, extubation time, presence of chest drains, and analgesic regimen prescribed on the day will initially be collected.

Then, the Ramsay sedation scale will be applied, and if the score presented by the patient is $\leq 3$ points, the instruments for assessing pain (at rest and cough stimulus) and cardiorespiratory parameters will be applied.

For pain assessment, first with the patient at rest, he will be asked about the presence of pain. If the answer is positive, you will be asked to indicate the location and assign a score for the intensity of your pain, from 0 to 10, according to the Numerical Scale, where 0 means no pain and 10 the worst pain ever felt. So, the pain will be classified as mild, moderate, or severe.

The patient will then be asked to rest their hands on their abdomen, inhale deeply and cough. You will be asked about the presence of pain when coughing and if the answer is positive, you will be asked to indicate the location and assign a score for the intensity of your pain, from 0 to 10 , according to the Numerical Scale, where 0 means absence of pain and 10 the worst pain ever felt. So, the pain will be classified as mild, moderate, or severe.

After measuring the cardiorespiratory parameters, the intervention will be applied (musical hearing for 20 minutes) for patients in the experimental group or standard care for patients in the control group.

After the intervention or standard care, the instruments for pain assessment (at rest and cough stimulus) will be applied again, according to the procedure described and the cardiorespiratory parameters.

Weekly, the research coordinator will check the consent terms and check the quality of the data collected in the instruments. After the conference by the coordinator, two researchers from the team will insert the data in an electronic bank stored in a password-protected computer, by double entry, to guarantee the

Page 10/22 
validation of the data. Only researchers will have access to the electronic database, collection instruments and consent terms.

The flowchart of the research participants is described in figure 1 and the steps of data collection are summarized in figure 2 .

\section{Security aspects}

To prevent cross-contamination, the team responsible for data collection will perform hand hygiene before and after the procedures; all equipment that comes into contact with the patient will be disinfected with $70 \%$ ethyl alcohol [39] and after each musical hearing, the disposable headphones protectors will be replaced.

During the pandemic period for the new coronavirus, all biosafety protocols will be strictly followed, and the data collection team will use all appropriate personal protective equipment, such as N95 mask, face shield, disposable apron, gloves and cap. Patients will also wear a disposable mask during collection in the pre- and postoperative period.

As for adverse events, their occurrence is not predictable in this research, given the nature of the intervention and the lack of description in the literature of adverse events resulting from musical hearing. However, in the event of any complication or adverse event to the participants, there will be a record of the information and immediate notification to the Research Ethics Committee.

\section{Evaluated outcomes}

\section{Primary outcome}

- Anxiety-state: refers to the average reduction (difference) of anxiety-state scores, obtained after subtracting the scores, before and after intervention or standard care, in both groups (experimental and control), preoperatively immediate.

\section{Secondary outcomes}

- Systolic blood pressure: refers to the mean reduction (difference) between the values of systolic blood pressure, obtained after subtracting the values, before and after intervention or standard care, in both groups (experimental and control), in the pre- immediate surgery and on the 1st postoperative day;

- Diastolic blood pressure: refers to the mean reduction (difference) between the values of diastolic blood pressure, obtained after subtracting the values, before and after intervention or standard care, in both groups (experimental and control), in the pre- immediate surgery and on the 1st postoperative day.

- Heart rate: refers to the average reduction (difference) between the heart rate values, obtained after subtracting the values, before and after intervention or standard care, in both groups (experimental and control), in the immediate preoperative period and on the 1st postoperative day; 
- Respiratory frequency: refers to the average of the reduction (difference) between the respiratory frequency values, obtained after subtracting the values, before and after intervention or standard care, in both groups (experimental and control), in the immediate preoperative period and on the 1st postoperative day;

- Oxygen saturation: refers to the average increase (difference) between the oxygen saturation values, obtained after subtracting the values, after and before intervention or standard care, in both groups (experimental and control), in the pre- immediate surgery and on the 1st postoperative day;

- Postoperative pain: refers to the mean reduction (difference) of pain scores at rest and to the cough stimulus, obtained after subtracting the scores, before and after intervention or standard care, in both groups (experimental and control), on the 1st postoperative day.

\section{Statistical methods}

\section{Sample size}

The sample size calculation was performed by a statistician without clinical involvement in the research and obtained, after conducting the pilot study, using the Power Analysis and Sample Size (PASS) version 13.0 software. It should be noted that the participants in the pilot study will not be part of the main study. A type I error $a=0.05$ and a type II error $\beta=0.2$ were considered, thus reaching a statistical power of $80 \%$.

For the pilot study, 13 patients were analyzed, being 07 patients from the control group and 06 from the experimental group. Considering anxiety as the primary outcome, the mean reduction in the STAI State scores for the experimental group was 5.67 points (standard deviation: 5.89) and for the control group it was 3.00 points (standard deviation: 7.30). Based on these values, the sample size calculated is 196 participants, that is, 98 per group.

The possible losses of participants will be compensated by the substitution by another participant in the same allocation group (intervention or control).

\section{Statistical analysis}

The collected data will be inserted in an Excel ${ }^{\circledR}$ spreadsheet of the Windows ${ }^{\circledR}$ program, using the double entry technique, for later validation. The identification of each study participant, as well as the respective allocation group, will be done through codes. Then, the validated database will be imported into the Statistical Package for the Social Sciences (SPSS) version 21.0 for processing and analysis.

The normality of the data will be verified by the Kolmogorov-Smirnov test or by the Shapiro-Wilk. To test the hypothesis of homogeneity of the two groups (experimental and control), it will be used, when applicable, the Student's t test for independent samples in quantitative variables and the chi-square test of homogeneity for categorical variables. 
The univariate exploratory analysis of the data will be performed by the distribution of absolute and relative frequency for categorical variables and measures of central tendency (mean and median) and of variability (amplitudes and standard deviation) for quantitative variables.

To compare the anxiety-state scores, cardiorespiratory parameters and pain intensity scores, at rest and to the cough stimulus, between the experimental and control groups, Student's $t$ test for independent samples will be used, when applicable.

To compare the anxiety-state scores, cardiorespiratory parameters and pain intensity scores, at rest and to the cough stimulus, within each group, experimental and control (intra-group analysis), a paired t-test will be used, when applicable.

Subgroup analysis will be performed and, if there is a violation of homogeneity of the groups for one or more confounding variables, a corresponding post hoc multivariate analysis will be performed.

To check the correlation between preoperative anxiety-state and intensity of postoperative pain, Pearson's correlation coefficient will be used, if applicable.

This research will consider a level of significance $a=5 \%$ and a confidence interval of $95 \%$. The data will be presented in tables and figures.

\section{Ethical aspects}

The study will be conducted in accordance with the ethical principles of the Declaration of Helsinki and Resolution No. 466, of December 12, 2012, of the National Health Council, Brazil, which deals with the regulatory guidelines and standards for research involving human beings [40].

The research project was registered and authorized by the Teaching and Research Management of HCUFTM and approved by the HC-UFTM Research Ethics Committee under Opinion No. 3,474,676, CAAE: 16210819.6.0000.8667. This research was registered on the Brazilian Clinical Trials Registry (ReBEC) platform under number RBR-8mdyhd (UTN: U1111-1238-1227).

Participants will be provided with all information regarding the research, such as objectives, procedures, risks, and benefits, as well as clarifications regarding voluntary participation and the possibility of withdrawing from the study at any time without prejudice to treatment. After reading the Clarification Term, the participants' signature on the Informed Consent Form will be obtained in two copies, one for the participant and one for the researcher.

To minimize the risk of loss of data confidentiality, participants will be identified, in the survey, by codes. At no time will personal data or any information that can identify participants be disclosed.

All documents generated as a result of the research, such as data collection instruments and terms, will be kept in a safe place, with restricted access and after the completion of the clinical trial, they will remain 
on file for five years and will subsequently be incinerated, under the responsibility of the coordinating researcher.

It should be noted that any necessary and significant modifications to the study protocol will be documented as an amendment to the protocol and should be formally reported to the Research Ethics Committee, the Brazilian Clinical Trials Registry platform, and the journal in which the protocol has been published.

\section{Dissemination of results}

The results of the study will be disseminated to the academic and professional community, through the release of public access to the thesis, the publication of scientific articles and the presentation of papers at scientific events.

\section{Discussion}

It is a protocol for a controlled, randomized clinical trial, with simple masking, which aims to evaluate the effect of musical hearing on preoperative state-anxiety, postoperative pain, at rest and coughing stimulus, and cardiorespiratory parameters in patients undergoing cardiac surgery. In view of the growing number of cardiac surgeries, the complexity of these procedures, the various complications that preoperative anxiety and pain after these surgeries can cause, and due to the need to evaluate and measure these phenomena, as well as proposing non-pharmacological interventions for his relief, this research is justified.

The study is feasible, safe, does not require invasive procedures and does not interfere with the treatment established for the participant. The intervention will be applied pre- and postoperatively through headphones containing a collection of classical music, pre-selected by the researchers, totalling 20 minutes and 28 seconds. The research is also relevant, due to the need for new studies to evaluate the effect of musical hearing in the context of cardiac surgery, since the results already presented were not conclusive, requiring more evidence.

One aspect that also deserves to be highlighted is the fact that the clinical trial evaluates the effect of musical hearing on pain during the cough stimulus and not only with the participant at rest, which allows a more comprehensive analysis of the intervention's effectiveness. However, the research has some limitations, such as: it does not allow the participant to choose the songs for the audition, which can interfere with the outcomes; the time of the hearing may not be enough to generate satisfactory results; the participants will not be masked, therefore, they will be aware of the receipt of the intervention and the history of the participants regarding treatment for anxiety and pain before hospitalization will not be considered.

It is hoped that the results of this clinical trial will provide evidence that will contribute to support the development and implementation of non-pharmacological interventions, specifically musical listening, in 
health services, to minimize anxiety and pain, which are considered relevant stressors for the surgical patient.

\section{Clinical trial status}

Study protocol version 2 of July 29, 2019. Recruitment of participants will start in October 2021 and the completion of this stage is expected to be October 2023.

\section{List Of Abbreviations}

CONSORT

Consolidated Standards of Reporting Trials; HC-UFTM:Hospital de Clínicas, Federal University of Triângulo Mineiro; STAl:State-Trait Anxiety Inventory; PASS:Power Analysis and Sample Size; ReBEC:Brazilian Registry of Clinical Trials; SPIRIT:Standard Protocol Items:Recommendations for Interventional Trials; SPSS:Statistical Package for the Social Sciences; UFTM:Federal University of Triângulo Mineiro; ICU:Intensive Care Unit.

\section{Declarations}

\section{Ethics approval and participation consent}

The research project was approved by the Research Ethics Committee of the Hospital de Clínicas, Federal University of Triângulo Mineiro under Opinion No. 3,474,676, CAAE: 16210819.6.0000.8667. Before the start of data collection, all participants will sign the Free and Informed Consent Form.

\section{Consent for publication}

Not applicable.

\section{Availability of data and materials}

Not applicable.

\section{Competing interests}

The authors declare that they have no competing interests.

\section{Funding}

There is no funding for this study.

\section{Authors' contributions}

MHB is the research coordinator. MHB and EVA structured the research design and developed the protocol. EVA will be responsible for allocating participants and implementing the intervention. VJH 
participated in the statistical design of the study and will contribute to the analysis of the data. MFF and MMSF will participate in the collection and organization of the data. MBGF, EB and PSP contributed to the critical review of the protocol and the preparation of the manuscript. All authors read and approved the final version of the manuscript.

\section{Acknowledgements}

Not applicable.

\section{References}

1. Kazitani BS, Furuya RK, Dantas RAS, Dessotte CAM. Preoperative anxiety and depression: differences among patients submitted to the first cardiac surgery. Rev Rene. 2018;19:e3079. http://dx.doi.org/10.15253/2175-6783.2018193079. Accessed 25 Aug 2018.

2. Santos MMB, Martins JCA, Oliveira LMN. Anxiety, depression and stress in the preoperative surgical patient. Rev Enf Ref. 2014;IV(3):7-15. http://dx.doi.org/10.12707/RIII1393. Accessed 01 Sep 2018.

3. Ribeiro KRA, Silva E. Preoperative anxiety before heart surgery: how can nursing act? Rev Soc Cardiol Estado de São Paulo. 2018;28(1):95-100. http://dx.doi.org/10.29381/0103-8559/2018280195-100. Accessed 02 Sep 2018.

4. Gonçalves KKN, Silva JI, Gomes ET, Pinheiro LLS, Figueiredo TR, Bezerra SMMS. Anxiety in the preoperative period of heart surgery. Rev Bras Enferm. 2016;69(2):397-403. http://dx.doi.org/10.1590/0034-7167.2016690225i. Accessed 28 Aug 2018.

5. Periañez CAH, Diaz MAC, Bonisson PLV, Simino GPR, Barbosa MH, De Mattia AL. Relationship of anxiety and preoperative depression with post-operative pain. Texto \& Contexto Enferm. 2020;29:e20180499. http://dx.doi.org/10.1590/1980-265X-TCE-2018-0499. Accessed 30 Dec 2020.

6. Bandeira RA, Gomes LO, Bezerra AJC, Duarte JA. Correlation between preoperative anxiety and acute postoperative pain in elderly patients submitted to transvesical prostatectomy. Rev Dor. 2017;18(4):291-7. https://doi.org/10.5935/1806-0013.20170118. Accessed 25 Aug 2018.

7. Robleda G, Sillero-Sillero A, Puig T, Gich I, Baños JE. Influence of preoperative emotional state on postoperative pain following orthopedic and trauma surgery. Rev Lat Am Enfermagem. 2014;22(5):785 - 91. https://doi.org/10.1590/0104-1169.0118.2481. Accessed 27 Aug 2018.

8. Andrade EV, Barbosa MH, Barichello E. Pain assessment in postoperative cardiac surgery. Acta Paul Enferm. 2010;23(2):224-9. https://doi.org/10.1590/S0103-21002010000200012. Accessed 25 Aug 2018.

9. Mello LC, Rosatti SFC, Hortense P. Assessment of pain during rest and during activities in the postoperative period of cardiac surgery. Rev Lat Am Enfermagem. 2014;22(1):136 - 43. https://doi.org/10.1590/0104-1169.3115.2391. Accessed 25 Aug 2018.

10. Menezes TC, Bassi D, Cavalcanti RC, Barros JE, Granja KS, Calles AC, et al. Comparisons and correlations of pain intensity and respiratory and peripheral muscle strength in the pre-and 
postoperative periods of cardiac surgery. Rev Bras Ter Intensiva. 2018;30(4):479 - 86. https://doi.org/10.5935/0103-507X.20180069. Accessed 27 Aug 2018.

11. Öğüt S, Sucu Dağ G. Pain characteristics and pain interference among patients undergoing open cardiac surgery. J Perianesth Nurs. 2019;34(4):757 - 66.

https://doi.org/10.1016/j.jopan.2018.10.009. Accessed 15 Dec 2019.

12. Roca J, Valero R, Gomar C. Localizaciones del dolor en el postoperatorio de cirugía cardiaca: cronología del dolor y respuesta al tratamiento. Rev Esp Anestesiol Reanim. 2017;64(7):391-400. https://doi.org/10.1016/j.redar.2017.01.002. Accessed 25 Aug 2018.

13. Chiapinotto S, Dallazen F, Bodnar ET, Winkelmann ER. Level of pain and functional independence of patients submitted to cardiac surgery. Rev Baiana Enferm. 2017;31(4):e21388.

http://dx.doi.org/10.18471/rbe.v31i4.21388. Accessed 28 Aug 2018.

14. Manworren RCB, Gordon DB, Montgomery R. Managing postoperative pain. Am J Nurs. 2018;118(1):36-43. http://dx.doi.org/10.1097/01.NAJ.0000529695.38192.67. Accessed 02 Jul 2019.

15. Chou R, Gordon DB, Leon-Casasola OA, Rosenberg JM, Bickler S, Brennan T, et al. Guidelines on the management of postoperative pain. J Pain. 2016;17(2):131 - 57. http://dx.doi.org/10.1016/j.jpain.2015.12.008. Accessed 25 Aug 2018.

16. Pinto Junior FEL, Ferraz DLM, Cunha EQ, Santos IRM, Batista MC. Influence of music on pain and anxiety due to surgery in patients with breast cancer. Rev Bras Cancerol. 2012;58(2):135 - 41. https://doi.org/10.32635/2176-9745.RBC.2012v58n2.611. Accessed 31 Aug 2018.

17. Jiménez-Jiménez M, García-Escalona A, Martín-López A, De Vera-Vera R, De Haro J. Intraoperative stress and anxiety reduction with music therapy: a controlled randomized clinical trial of efficacy and safety. J Vasc Nurs. 2013;31(3):101-6. https://doi.org/10.1016/j.jvn.2012.10.002. Accessed 26 Aug 2018.

18. Forooghy M, Tabrizi EM, Hajizadeh E, Pishgoo B. Effect of music therapy on patients' anxiety and hemodynamic parameters during coronary angioplasty: a randomized controlled trial. Nurs Midwifery Stud. 2015;4(2):e25800. http://dx.doi.org/10.17795/nmsjournal25800. Accessed 08 Mar 2019.

19. Franzoi MAH, Goulart CB, Lara EO, Martins G. Music listening for anxiety relief in children in the preoperative period: a randomized clinical trial. Rev Lat Am Enfermagem. 2016;24:e2841. http://dx.doi. org/10.1590/1518-8345.1121.2841. Accessed 03 Sep 2018.

20. Akbas A, Gulpinar MT, Sancak EB, Karakan T, Demirbas A, Utangac MM, et al. The effect of music therapy during shockwave lithotripsy on patient relaxation, anxiety, and pain perception. Ren Fail. 2016;38(1):46 - 9. https://doi.org/10.3109/0886022X.2015.1096728. Accessed 05 Nov 2019.

21. Hsu KC, Chen LF, Hsiep PH. Effect of music intervention on burn patients' pain and anxiety during dressing changes. Burns. 2016;42(8):1789-96. https://doi.org/10.1016/j.burns.2016.05.006. Accessed 05 Nov 2019. 
22. Özer N, Özlü ZK, Arslan S, Günes N. Effect of music on postoperative pain and physiologic parameters of patients after open heart surgery. Pain Manag Nurs. 2013;14(1):20 - 8. https://doi.org/10.1016/j.pmn.2010.05.002. Accessed 08 Mar 2019.

23. Ajorpaz NM, Mohammadi A, Najaran H, Khazaei S. Effect of music on postoperative pain in patients under open heart surgery. Nurs Midwifery Stud. 2014;3(3):e20213. https://dx.doi.org/10.17795/nmsjournal20213. Accessed 08 Mar 2019.

24. Koelsch S, Jäncke L. Music and the heart. Eur Heart J. 2015;36(44):3043-9. https://doi.org/10.1093/eurheartj/ehv430. Accessed 05 Nov 2019.

25. Grafton-Clarke C, Grace L, Harky A. Music therapy following cardiac surgery: is it an effective method to reduce pain and anxiety? Interact Cardiovasc Thorac Surg. 2019;28(5):722-7. https://doi.org/10.1093/icvts/ivy311. Accessed 01 Nov 2019.

26. Boutron I, Altman DG, Moher D, Schulz KF, Ravaud P. CONSORT statement for randomized trials of nonpharmacologic treatments: A 2017 update and a CONSORT extension for nonpharmacologic trial abstracts. Ann Intern Med. 2017;167(1):40 - 7. https://doi.org/10.7326/M17-0046. Accessed 19 Feb 2019.

27. Chan AW, Tetzlaff JM, Altman DG, Laupacis A, Gøtzsche PC, Krleža-Jerić K, et al. SPIRIT 2013 statement: defining standard protocol items for clinical trials. Ann Intern Med. 2013;158(3):200-7. https://doi.org/10.7326/0003-4819-158-3-201302050-00583. Accessed 19 Feb 2019.

28. Joanna Briggs Institute. The Joanna Briggs Institute Best Practice Information Sheet: music as an intervention in hospitals. Nurs Health Sci. 2011;13(1):99-102. https://doi.org/10.1111/j.14422018.2011.00583.x. Accessed 07 Sep 2019.

29. Silva KF, Felix MM, Cruz LF, Barichello E, Pires PS, Mattia AL, et al. Effects of music on the anxiety of blood donors: randomized clinical trial. Acta Paul Enferm. 2021;34;eAPE00461. https://doi.org/10.37689/acta-ape/2021ao00461. Accessed 24 Mar 2021.

30. Krout RE. Music listening to facilitate and promote wellness: Integrated aspects of our neurophysiological responses to music. Arts Psychother. 2007;34(2):134 - 41. https://doi.org/10.1016/j.aip.2006.11.001. Accessed 16 Feb 2019.

31. American Society of Anesthesiologists. ASA physical status classification system. 2020. https://www.asahq.org/standards-and-guidelines/asa-physical-status-classification-system. Accessed 05 Jan 2021.

32. Spielberger CD, Gorsuch RL, Lushene RE. Manual for the State-Trait Anxiety Inventory. Palo Alto: Consulting Psychologists Press; 1970.

33. Spielberger CD, Gorsuch RL. Lushene RE. Inventário de Ansiedade Traço-Estado (IDATE). Biaggio AMB, Natalício L. translators. Rio de Janeiro: CEPA; 1979.

34. Biaggio AMB, Natalício L, Spielberger CD. Desenvolvimento da forma experimental em português do Inventário de Ansiedade Traço-Estado (IDATE) de Spielberger. Arq Bras Psicol Apl. 1977;29(3):31-44. http://bibliotecadigital.fgv.br/ojs/index.php/abpa/article/view/17827/16571. Accessed 31 Jan 2019. 
35. Kaipper MB. Avaliação do inventário de ansiedade traço-estado (IDATE) através da análise de Rasch [thesis]. Porto Alegre:Universidade Federal do Rio Grande do Sul;2008.138p. http://hdl.handle.net/10183/17463. Accessed 10 Aug 2019.

36. Barroso WKS, Rodrigues CIS, Bortolotto LA, Mota-Gomes MA, Brandão AA, Feitosa ADM, et al. Brazilian Guidelines of Hypertension - 2020. Arq Bras Cardiol. 2020; [online].ahead print. http://abccardiol.org/article/diretrizes-brasileiras-de-hipertensao-arterial-2020/. Accessed 04 Jan 2021.

37. Sousa FAEF. Dor: o quinto sinal vital. Rev Lat Am Enfermagem. 2002;10(3):446-7. https://doi.org/10.1590/S0104-11692002000300020. Accessed 01 Feb 2019.

38. Calil AM, Pimenta CAM. Pain intensity and adequacy of analgesia. Rev Lat Am Enfermagem. 2005;13(5):692-9. https://doi.org/10.1590/S0104-11692005000500013. Accessed 01 Feb 2019.

39. Brasil. Ministério da Saúde. Agência Nacional de Vigilância Sanitária. Resolução nº 15, de 15 de março de 2012. Dispõe sobre requisitos de boas práticas para o processamento de produtos para saúde e dá outras providências. Brasília: Diário Oficial da União; 2012.

https://bvsms.saude.gov.br/bvs/saudelegis/anvisa/2012/rdc0015_15_03_2012.html. Accessed 07 Sep 2019.

40. Brasil. Ministério da Saúde. Conselho Nacional de Saúde. Resolução n 466 , de 12 de dezembro de 2012. Dispõe sobre diretrizes e normas regulamentadoras de pesquisas envolvendo seres humanos. Brasília: Diário Oficial da União; 2013.

https://bvsms.saude.gov.br/bvs/saudelegis/cns/2013/res0466_12_12_2012.html. Accessed 07 Sep 2019.

\section{Figures}




\begin{tabular}{|c|c|c|c|c|c|c|c|c|}
\hline & \multicolumn{8}{|c|}{ STUDY PERIOD } \\
\hline & \multicolumn{5}{|c|}{ Preoperative } & \multicolumn{3}{|c|}{ Postoperative } \\
\hline & \multirow{3}{*}{$\begin{array}{c}\text { Enrolment } \\
-t_{1}\end{array}$} & \multirow{3}{*}{$\begin{array}{c}\text { Allocation } \\
0\end{array}$} & \multicolumn{5}{|c|}{ Post-allocation } & \multirow{2}{*}{$\begin{array}{c}\begin{array}{c}\text { Close- } \\
\text { out }\end{array} \\
t_{6} \\
\text { After }\end{array}$} \\
\hline TIMEPOINT & & & $\begin{array}{c}t_{1} \\
\text { Before }\end{array}$ & $\begin{array}{c}\boldsymbol{t}_{\mathbf{2}} \\
\text { Intervention } \\
\text { or standard } \\
\text { care }\end{array}$ & $\begin{array}{r}\boldsymbol{t}_{3} \\
\text { After }\end{array}$ & $\begin{array}{c}\boldsymbol{t}_{\mathbf{4}} \\
\text { Before }\end{array}$ & $\begin{array}{c}\boldsymbol{t}_{\mathbf{5}} \\
\text { Intervention } \\
\text { or standard } \\
\text { care }\end{array}$ & \\
\hline & & & \multicolumn{3}{|c|}{$\begin{array}{c}\text { Up to } 24 \text { hours before } \\
\text { surgery }\end{array}$} & \multicolumn{3}{|c|}{24 to 48 hours after surgery } \\
\hline \multicolumn{9}{|l|}{ ENROLMENT: } \\
\hline Eligibility screen & $\mathrm{x}$ & & & & & & & \\
\hline $\begin{array}{l}\text { Reading the } \\
\text { clarification term }\end{array}$ & $\mathrm{x}$ & & & & & & & \\
\hline Informed consent & $\mathrm{x}$ & & & & & & & \\
\hline Allocation & & $\mathrm{x}$ & & & & & & \\
\hline \multicolumn{9}{|l|}{ INTERVENTIONS: } \\
\hline Listening to music & & & & $\mathrm{x}$ & & & $\mathrm{x}$ & \\
\hline Standard care & & & & $x$ & & & $\mathrm{x}$ & \\
\hline \multicolumn{9}{|l|}{ ASSESSMENTS: } \\
\hline $\begin{array}{l}\text { Anxiety (STAI } \\
\text { State score) }\end{array}$ & & & $\mathrm{x}$ & & $x$ & & & \\
\hline $\begin{array}{l}\text { Cardiorespiratory } \\
\text { parameters }\end{array}$ & & & $\mathrm{x}$ & & $\mathrm{x}$ & $\mathrm{x}$ & & $\mathrm{x}$ \\
\hline $\begin{array}{l}\text { Pain (Numerical } \\
\text { Scale score) }\end{array}$ & & & & & & $\mathrm{X}$ & & $\mathrm{X}$ \\
\hline
\end{tabular}

Figure 1

Standard Protocol Items: Recommendations for Interventional Trials (SPIRIT) flowchart. 


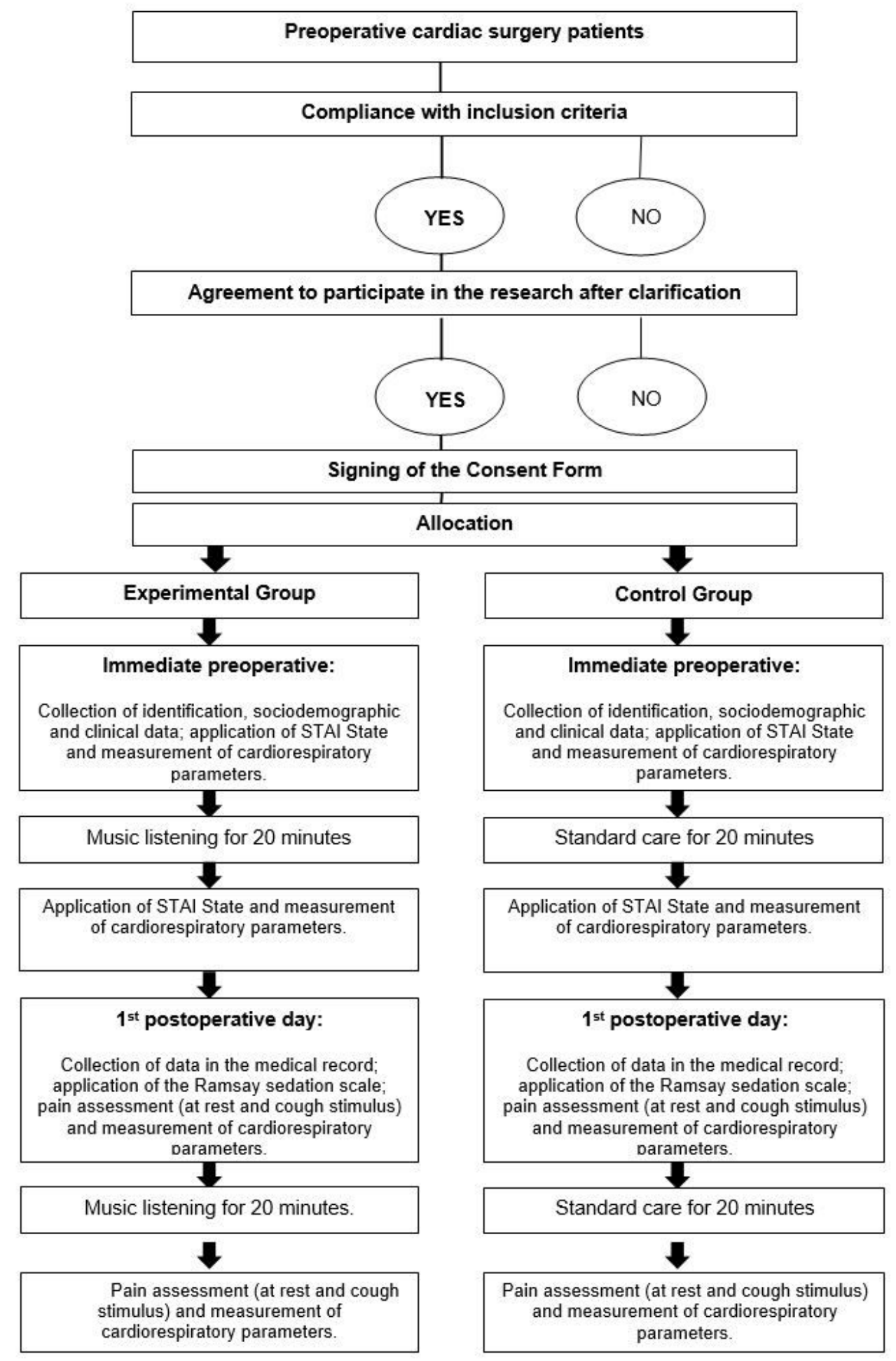

Figure 2

Diagram of the steps of data collection.

\section{Supplementary Files}

This is a list of supplementary files associated with this preprint. Click to download. 
- Additionalfile1checklistSPIRIT.doc 